\title{
Towards a New Set of High-Precision Radial-Velocity Standard Stars
}

\author{
S. Udry, M. Mayor and D. Queloz ${ }^{1}$ \\ Observatoire de Genève, ch. des Maillettes 51,CH-1290 Sauverny
}

\begin{abstract}
CORAVEL photoelectric radial-velocity measurements associated with the high precision achieved by the ELODIE spectrograph on a fair time span allow us to propose a new list of highprecision radial-velocity standards with velocity variations lower than a few times $10 \mathrm{~m} \mathrm{~s}^{-1}$.
\end{abstract}

\section{Introduction}

To rely on the results provided by a spectrograph on a long term basis, a quality control of the temporal evolution of the obtained results is needed. This is generally done by using a set of regularly followed standard stars that allows you to correct for instrumental effects. In their presentation, Stefanik, Latham \& Torres (1999) nicely recalled the long term historical common effort undertaken with instruments achieving precisions of a few times $100 \mathrm{~m} \mathrm{~s}^{-1}$ (CORAVEL, $\mathrm{CfA}, \mathrm{DAO}, \ldots)$ to provide a good list of reliable radial-velocity standards for the astronomical community, cleaning the initially selected sample from candidates with intrinsic or orbital radial-velocity variations (see Udry et al. 1999 for results on the CORAVEL standard star sample).

Since about a decade, the precision achieved in measuring radial velocities has drastically improved. Because of the time base required for a star to be recognized as a standard, the technical evolution of these last years leads now, with a little delay in terms of standard stars, to a transition point between moderate- and high-precision $\left(\leq 10 \mathrm{~m} \mathrm{~s}^{-1}\right)$ radial-velocity measurements, as achieved for example at Lick, Keck, McDonald or with AFOE, ELODIE or CORALIE. The surveys with the latter facilities are now old enough to allow us to define a set of standard stars, at the new available high precision, taking nevertheless advantage of the very long period of continuous efforts carried on with the instruments of moderate precision.

In this contribution, we will focus on the two instruments intensively used by our group in Geneva, namely the CORAVEL and ELODIE spectrometers, for which we have a large number of measurements at our disposal. The 20 years of CORAVEL measurements, associated with the high precision achieved with ELODIE on a fair time span provide the perfect tool for proposing a new list of high-precision radial-velocity standards.

\footnotetext{
${ }^{1}$ also at JPL, 4800 Oak Grove Drive, Pasadena, CA-91109
} 


\section{Radial-velocity standards and intrinsic stellar variability}

Before combining the CORAVEL and ELODIE samples, we have to define the properties required for the future set of standard stars. The first prerequisite is of course the radial-velocity non-variability at the desired precision level.

As defined several decades ago, the present list of IAU standard stars mainly includes very bright stars the majority of which being giants and supergiants. If some of those stars seem to be constant at a moderate precision ${ }^{1}$ level (a few 100 $\mathrm{m} \mathrm{s}^{-1}$ ), all giant and supergiant stars are now known to present intrinsic radialvelocity variations on short time scales at a $10 \mathrm{~m} \mathrm{~s}^{-1}$ precision level (pulsation, spots, activity). The level of intrinsic variations of a particular star will thus fix the precision level of the radial-velocity standard, whatever the number of measurements defining the mean velocity value. As a consequence, giant and supergiant stars (more generally evolved stars) have to be avoided when defining a list of high-precision radial-velocity standards. We have to use dwarfs.

To accommodate the different types of programs requiring radial-velocity standards, the list of standard stars should cover as much as possible a wide range of magnitude, color and velocity values as well as providing a full coverage of the sky (season and hemisphere). These requirements are still difficult to fulfill, there are not high-precision surveys for all type of stars yet. However, the large on-going ELODIE planet-search program already provides good G-K candidates, brighter than $m_{V}=8$, for the northern sky.

\section{The CORAVEL and ELODIE systems}

To combine the results coming from two spectrographs, we have to compare and link the two instruments in a same velocity reference system. In our case, the reference system will be ELODIE, less sensitive to systematic effects, as shown below. The instruments have first to prove their stability on a long term basis so we can trust the results and then will be able to confidently correct for possible systematic effects between them. In the next section, we recall some of the features of the two spectrographs, emphasizing their differences.

\subsection{Instrumental characteristics}

CORAVEL is an échelle spectrograph with a built-in cross correlation mechanism with a physical template located in the focal plane of the spectrograph (Baranne et al. 1979). The holes in the template were designed to exactly match the absorption lines of Arcturus (K2III) at given conditions of pressure and temperature. Changes of these conditions induce changes of the refraction index of the air and the absorption line positions will no more exactly correspond to the template windows. The effect of the pressure change is also to shear the star spectrum in the focal plane enhancing the effect. Finally, the difference in position between the absorption lines and the mask holes within an order is a function $\left(f\left(\lambda, V_{r}, B-V\right)\right)$ of the wavelength and of the radial velocity

\footnotetext{
${ }^{1}$ In the course of the time, the sample has been cleaned from stars with large-amplitude intrinsic variations and from previously unknown binary systems
} 
Table 1. CORAVEL and ELODIE spectrograph characteristics

\begin{tabular}{lll}
\hline Spectrograph & CORAVEL & ELODIE \\
Spectrum & échelle & échelle \\
$\sharp$ orders & 20 & 67 \\
Resolution & 20000 & 42000 \\
$V_{r}$ reference & Iron lamp & ThAr lamp \\
$V_{r}$ estimate & Physically built-in cross- & Numerical cross-correlation \\
& correlation mechanism & \\
& (incidence angle variation) & $(\lambda$-solution with ThAr) \\
Systematic effects & $V_{r}, B-V$ & no \\
\hline
\end{tabular}

and spectral type of the observed star. This effect called mismatch is especially significant at the order edges. Furthermore, the spectral shift is proportional to the velocity amplitude, the mismatch effect will then be larger for high velocity stars. See Baranne, Mayor, \& Poncet (1979) for further details and quantitative estimates of the mismatch. The first-order effects are taken into account by the use of a calibration iron lamp before and after a stellar observation. Secondorder effects result in systematic effects depending mainly on the star color and velocity.

With ELODIE the high-resolution stellar and ThAr calibration spectra are recorded on a CCD. The ThAr emission lines are used to derive a wavelength solution on the CCD and locate the stellar lines within $20 \mathrm{~m} \mathrm{~s}^{-1}$. The redressed spectrum is then numerically cross-correlated with a binary $([0,1])$ template suited for the considered type of star. As the absorption lines are accurately located by the ThAr wavelength calibration, no mismatch occurs. Moreover, systematic effects due to temperature variations are minimized by temperature controlling the spectrograph (and spectrograph room) and in such a controlled environment pressure effects remain very small.

\subsection{Instrumental stability}

Before joining the CORAVEL and ELODIE data by quantifying and correcting for the differences between the 2 instruments using a set of link stars, we have to verify the quality of the results on a long term basis.

For CORAVEL, instrumental drifts up to more than $1 \mathrm{~km} \mathrm{~s}^{-1}$ are observed on timescales of 2-3 years. This is illustrated in Fig. 1 left (upper panel) for the southern CORAVEL installed on the 1.54-m Danish telescope at La Silla till the end of 1997. The causes of the variations are unknown and seem not correlated with temperature and pressure (seasonal features). A set of about 50 CORAVEL standard stars regularly followed, whose radial-velocity variations are displayed in the figure, are used to correct for these instrumental drifts. Zero-point shifts due to work on the instrument are corrected as well. The result of the correction procedure is shown in the lower panel of the figure for the same set of stars.

The continuous effort on the CORAVEL standard stars is illustrated by the number of measurements and span distributions given in Fig. 1 of Udry et al. (1999). A precision of about $0.3 \mathrm{~km} \mathrm{~s}^{-1}$ is thus guaranteed over more than 18 

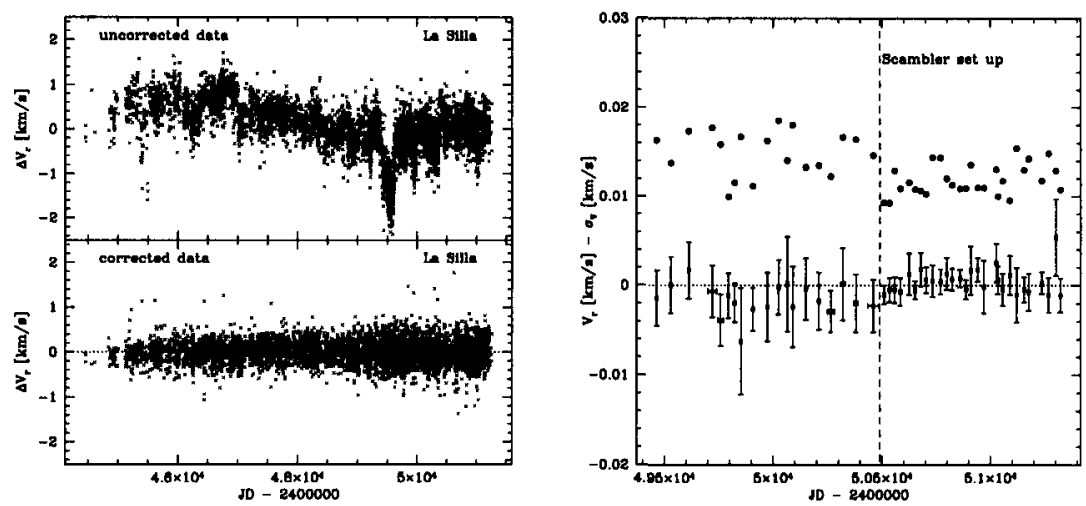

Figure 1. Left. Temporal variation of the southern CORAVEL zeropoint defined by a set of standard stars before (upper panel) and after (lower panel) correction. Right. Mean radial-velocity variations per observing run of the constant stars of the ELODIE planet-search program (squares). The mean value stays well below the $10 \mathrm{~m} \mathrm{~s}^{-1}$ limit with a dispersion around $10-15 \mathrm{~m} \mathrm{~s}^{-1}$ (circles)

years for the southern CORAVEL and, in the same way, for more than 20 years for the northern CORAVEL on the 1-m Swiss telescope at the Observatoire de Haute-Provence. Moreover, the uniform distributions of the $\chi^{2}$ probability of constant stars of the CORAVEL radial-velocity HIPPARCOS programs (Fig. 4 of Udry et al. 1997) also indicate that the CORAVEL errors are very well controlled.

For ELODIE, the subset of radial-velocity constant stars of the planetsearch program $\left(P\left(\chi^{2}\right)>0.01\right.$ and $\left.N \geq 3\right)$ are used as standards to estimate the zero-point variations of the instrument. As shown in Fig. 1 right, these variations stay below the $5 \mathrm{~m} \mathrm{~s}^{-1}$ level over the full 4 years of the survey, even improving with the introduction of a double scrambler device in 1996.

\subsection{From CORAVEL to ELODIE}

We shall now establish the velocity-color corrections needed to go from the CORAVEL radial-velocity system to the ELODIE one chosen to be the reference. This will be done in two steps. First the raw data coming from the northern CORAVEL are linked to ELODIE and then the raw data from the southern CORAVEL are linked to the "new" northern CORAVEL system. There is no efficient direct link between the southern CORAVEL and ELODIE because the sample of stars observed with the two instruments is too small.

From the northern CORAVEL to ELODIE. From the CORAVEL and ELODIE databases, suitable constant stars with large numbers of radial-velocity measurements and observational spans are selected: $N_{C O R} \geq 5,(\Delta T)_{C O R}>5 \mathrm{yr}$, $(\Delta T)_{E L O}>1 \mathrm{yr}$, and $P\left(\chi^{2}\right) \geq 0.01$ for both instruments. 149 stars coming from different non-disjoint programs fulfill our strong criteria: 


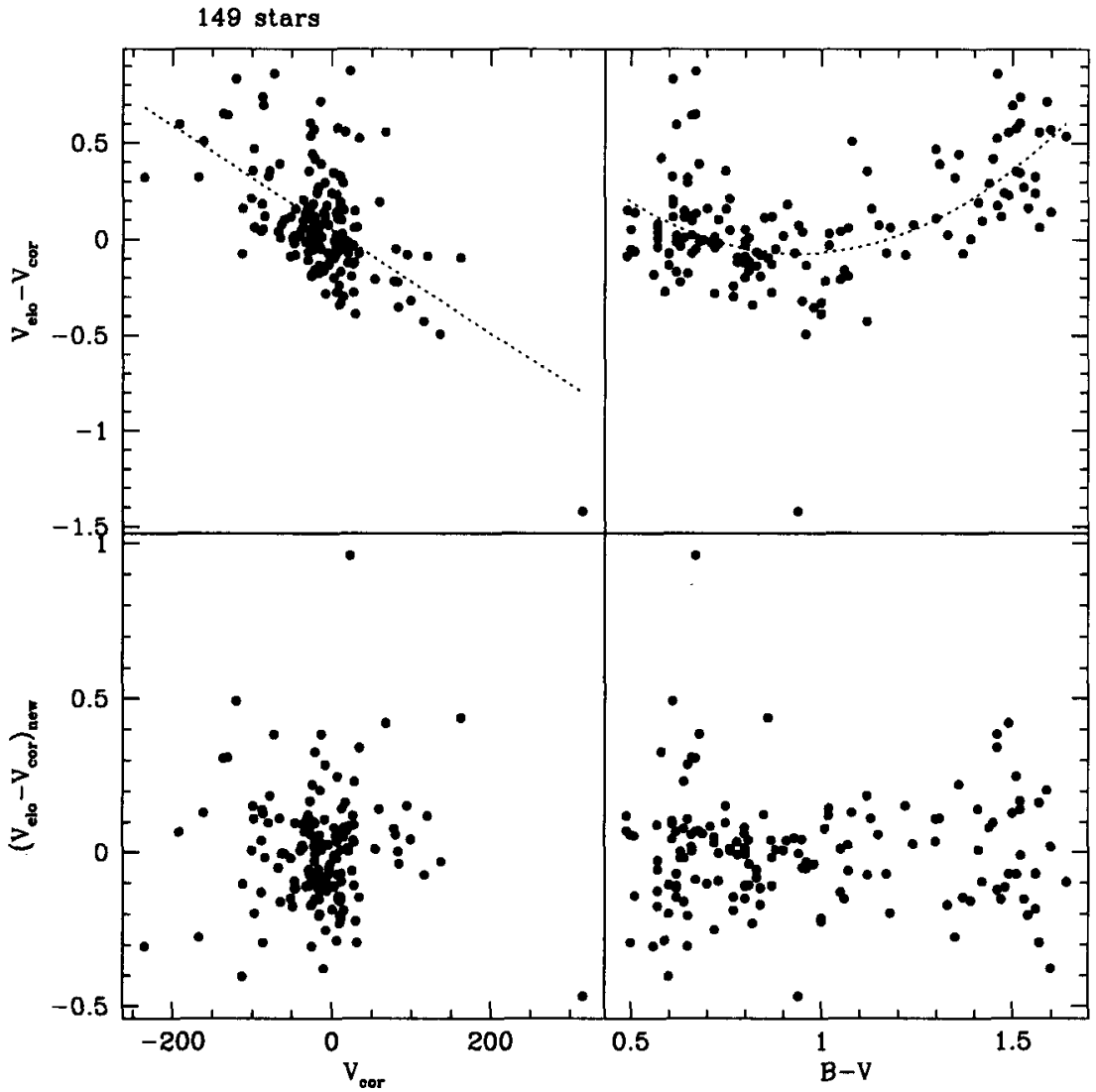

Figure 2. Upper panels. Differences between raw radial velocities coming from CORAVEL (north) and ELODIE as a function of color and velocity amplitude. Lower panels. Same but with the CORAVEL data in the new "ELODIE" system

- a subsample of the CORAVEL standards (19 stars),

- a subset of the ELODIE planet-search survey of solar-type stars (84 stars),

- a subset of the ELODIE M-dwarf binary survey to extend the sample towards redder stars (Delfosse et al. 1999; 29 stars),

- a set of high-velocity stars measured on purpose (31 stars).

The good color-velocity coverage of the sample allows us to point out the linear dependency in velocity and the quadratic dependency in color of the observed velocity difference between the two instruments (Fig. 2). A least-squared fit of the function

$$
V_{E L O}-V_{C O R N}=a_{0}+a_{1} V_{C O R N}+a_{2}(B-V)+a_{3}(B-V)^{2}
$$


with $V_{C O R N}$ the northern CORAVEL raw radial velocity, gives the following solution:

$$
\begin{array}{llll}
a_{0}= & 0.923 & \pm & 0.171 \\
a_{1}= & 2.72 \cdot 10^{-3} & \pm & 2.39 \cdot 10^{-4} \\
a_{2}= & -2.236 & \pm & 0.355 \\
a_{3}= & 1.23 & \pm & 0.167 .
\end{array}
$$

For our selected sample, the observed scattering of the CORAVEL radial velocities is decreasing from $0.3 \mathrm{~km} \mathrm{~s}^{-1}$ in the "old" historical system down to $0.18 \mathrm{~km} \mathrm{~s}^{-1}$ after the correction. We also have checked that, in the new "ELODIE" system, no other dependency on stellar parameters $(v \sin i,[\mathrm{Fe} / \mathrm{H}])$ subsists.

CORAVEL: from south to north. Historically and for various reasons, among the two CORAVELs, the southern one was chosen to be the reference but, as we shall see in the following, this was not such a good idea because in the southern sky, to be able to measure velocities in the Magellanic Clouds $\left(\sim 300 \mathrm{~km} \mathrm{~s}^{-1}\right)$, the zero-shift point of the instrument was set to $100 \mathrm{~km} \mathrm{~s}^{-1}$ contrarily to the northern CORAVEL for which the zero-shift point was set to $0 \mathrm{~km} \mathrm{~s}^{-1}$. This difference introduces mismatch differences for a same star observed with the two instruments and thus velocity differences being a function of the star color and velocity amplitude.

Among the large number of stars observed with the two CORAVELs (adequate declination range), 301 stars well observed over a long time span were selected in order to get a good color-velocity plane coverage. In addition to the same sources as in the previous case, these stars were also taken from:

- the CORAVELs' large G-K-M binary surveys in both hemispheres, database.

- a subset of very high velocity stars selected on purpose in the CORAVEL

Figure 3 shows a linear dependency in color and a quadratic dependency in velocity of the radial-velocity differences between the two CORAVELs for the selected sample. A least-squared fit of the function

$$
V_{C O R}-V_{C O R S}=a_{0}+a_{1} V_{C O R S}+a_{2} V_{C O R S}^{2}+a_{3}(B-V)
$$

with $V_{C O R}$ the CORAVEL radial velocity in the new "ELODIE" system and $V_{C O R S}$ the southern CORAVEL raw radial velocity, yields the following solution:

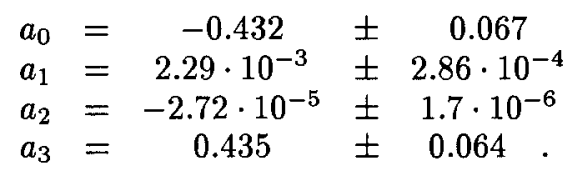

The observed scattering of the radial velocities of the sample (mainly due to velocity effects) is decreased from $0.55 \mathrm{~km} \mathrm{~s}^{-1}$ down to $0.36 \mathrm{~km} \mathrm{~s}^{-1}$ in the new "ELODIE" system. As previously we also have checked that no other dependency on stellar parameters $(v \sin i,[\mathrm{Fe} / \mathrm{H}])$ subsists in the new system.

The link equations derived in the previous subsections allow us to transform all the raw CORAVEL data into velocities in the new "ELODIE" system. From 


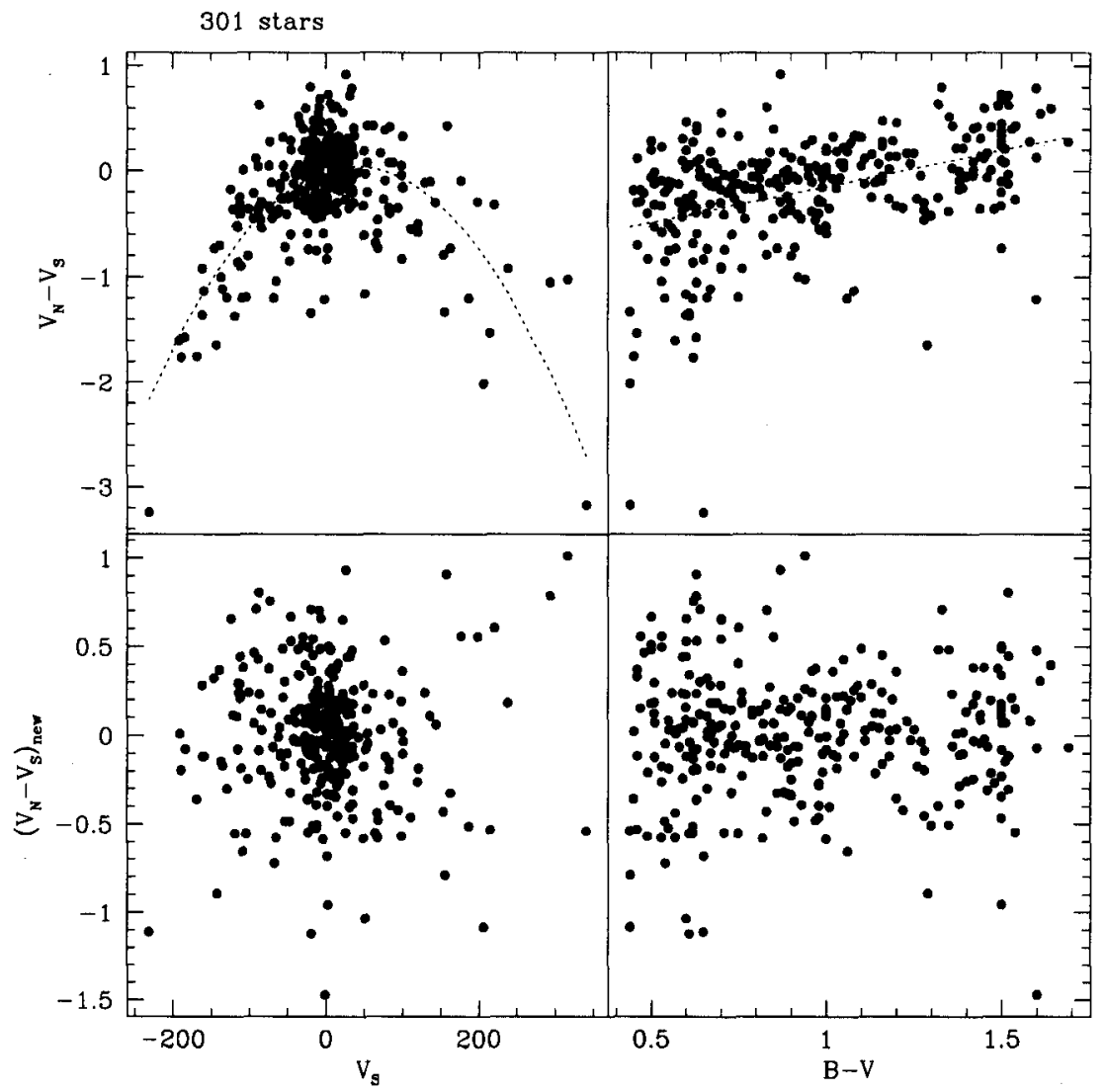

Figure 3. Upper panels. Differences between raw radial velocities coming from southern CORAVEL $\left(V_{S}\right)$ and northern CORAVEL velocities in the ELODIE system $\left(V_{N}\right)$ as a function of color and velocity amplitude. Lower panels. Same but with all the data in the new "ELODIE" system

now on (i.e. from January 1st 2000), the CORAVEL radial velocities will be expressed in the new system.

It should be noted here that raw CORAVEL data do not correspond to "old" published CORAVEL velocities and thus the given equations cannot be applied to the previously published data to change the velocity reference system.

CORAVEL-CfA discrepancy. The large differences observed between data from the two CORAVELs enlighten the long standing question of the difference between CORAVEL and CfA results. Part of the discrepancy came from the choice of the southern CORAVEL, more sensitive to mismatch (see above), as reference system. However, as shown in Fig. 4, this is not the end of the story. If 

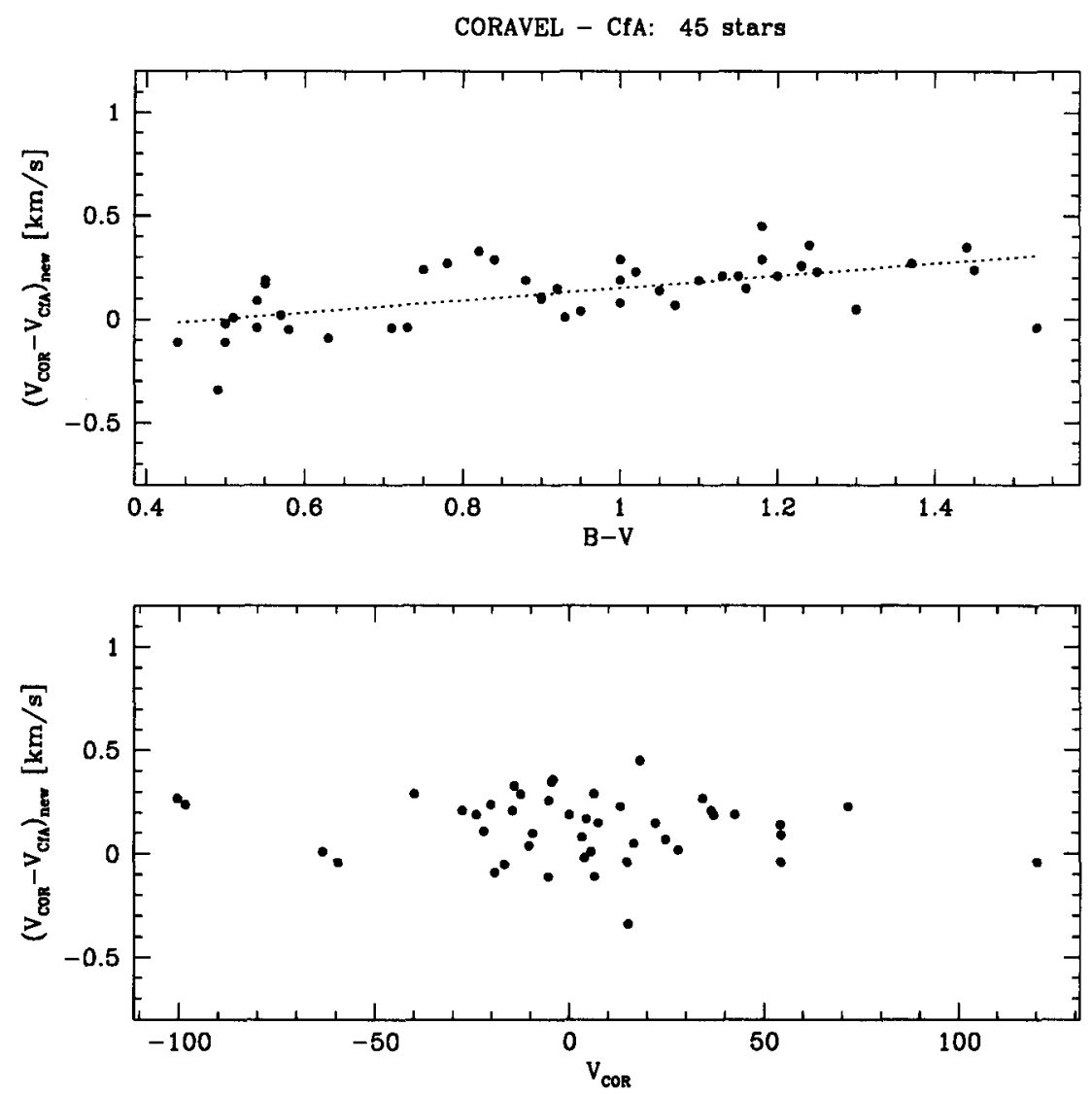

Figure 4. Differences between new CORAVEL and CfA radial velocities as a function of color (upper panel) and velocity amplitude (lower panel)

the velocity dependency has been removed, about half of the color effect is still remaining. Origins of the subsisting difference have probably to be sought in the line selection (narrow vs broad lines, convection effect, etc.) for the crosscorrelation templates (in both methods) that may introduce velocity zero-point shifts. The very small wavelength window used in the CfA technique to derive their radial velocities probably also plays a role.

\section{A new set of high-precision radial-velocity standards}

From the best available ELODIE data, namely the planet-search program initiated in 1994 by Mayor and Queloz (1995), we have selected 38 constant stars to be proposed as high-precision radial-velocity standards (Table 2). They fulfill 
the following strong criteria: $N_{E L O} \geq 5, \Delta T_{E L O} \geq 1000 \mathrm{~d}, N_{C O R} \geq 9, \Delta T_{C O R} \geq$ $2500 \mathrm{~d}$ and $P\left(\chi^{2}\right)>0.01$ for both instruments. Whereas the ELODIE-related criteria insure the star's non-variability, the long CORAVEL time coverage is mainly needed to avoid eccentric orbits. The actual $N$ and $\Delta T$ parameters of the stars are given in the table as well.

The ELODIE instrument insures a temporal variability level below $15 \mathrm{~m}$ $\mathrm{s}^{-1}$ for these stars. However, because of the relative notion of absolute velocity (Einstein effect, convection effect, narrow/broad lines selection for the template definition, etc; see several contributions in this volume), the velocities in the table are given rounding off the values at a $50 \mathrm{~m} \mathrm{~s}^{-1}$ precision level.

The list of proposed standards covers a fair range in visual magnitudes from 4.27 to 7.64 . Some high-velocity stars are however still missing. The number of selected standards is a function of our ad hoc criteria on the CORAVEL data, mainly $N_{S T D}=f\left(N_{C O R}, \Delta T_{C O R}\right)$. For special needs, more candidates could be added to the list by just relaxing a little bit these criteria. Moreover, with passing time, new candidates will naturally come out of the on-going highprecision planet-search and binary surveys. This will be especially interesting for redder stars (M-dwarf survey) and in the southern sky (CORALIE planet-search program).

In the future, the proposed standard stars will be followed with ELODIE to further insure their long term non-variability. For comparison purposes, the next step will then be to link the ELODIE system to the more general reference system defined by the motion of minor planets.

Our evolutive list of high-precision standard stars will be available on our dedicated web page at the following address:

$$
\text { http://obswww.unige.ch/ udry/std/std.html }
$$

Acknowledgments. We are grateful to the CORAVEL and ELODIE technical support teams for their efficient work and to the Fonds National Suisse de la Recherche Scientifique for its continuous financial support.

\section{References}

Baranne, A., Mayor, M., \& Poncet, J.-L. 1979, Vistas in Astronomy, 23, 279

Delfosse, X., Forveille, T., Beuzit, J.-L., Udry, S., Mayor, M., \& Perrier, C. 1999, A\&A, 344, 897

Mayor, M., \& Queloz, D. 1995, Nature, 378, 355

Stefanik, R.P., Latham, D.W., \& Torres, G. 1999, these Proceedings

Udry, S. et al. (13 authors) 1997, in Hipparcos Venice'97 (ESA SP-402), B. Battrick, Noordwijk: European Space Agency, 693

Udry, S., Mayor, M., Maurice, E., Andersen, J., Imbert, M., Lindgren, H., Mermilliod, J.-C., Nordström, B., \& Prévot, L. 1999, these Proceedings 
Table 2. Proposed list of high-precision radial-velocity standards

\begin{tabular}{|c|c|c|c|c|c|c|c|}
\hline HD & $m_{V}$ & $\bar{B}-V$ & $\begin{array}{c}V_{r} \\
{\left[\mathrm{~km} \mathrm{~s}^{-1}\right]}\end{array}$ & $N_{\mathrm{ELO}}$ & $\begin{array}{r}\Delta T_{\text {ELO }} \\
\text { [days] }\end{array}$ & $N_{\mathrm{COR}}$ & $\begin{array}{r}\Delta T_{\mathrm{COR}} \\
\text { [days] }\end{array}$ \\
\hline 3765 & 7.36 & 0.94 & -63.30 & 17 & 1161.8 & $\overline{176}$ & 7476 \\
\hline 10780 & 5.63 & 0.80 & 2.70 & 26 & 1155.8 & 14 & 4711 \\
\hline 32923 & 60 & 0.66 & 20.50 & 16 & 1277.7 & 30 & 5100 \\
\hline 38230 & 7.36 & 0.83 & -29.25 & 12 & 1160.8 & 14 & 3140 \\
\hline 42807 & 6.45 & 0.66 & 6.00 & 13 & 1152.8 & 28 & 5931 \\
\hline 50692 & 5.74 & 0.57 & -15.05 & 15 & 1331.2 & 15 & 2652 \\
\hline 62613 & 6.56 & 0.72 & -7.85 & 16 & 1477.0 & 9 & 3304 \\
\hline 65583 & 7.00 & 0.72 & 14.80 & 10 & 1159.0 & 132 & 6940 \\
\hline 73667 & 7.64 & 0.83 & -12.10 & 10 & 1213.8 & 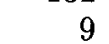 & 3591 \\
\hline 79210 & 7.62 & 1.41 & 10.65 & 14 & 1475.0 & 21 & 6996 \\
\hline 82106 & 7.20 & 1.00 & 29.75 & 14 & 1156.9 & 10 & 3694 \\
\hline 82885 & : & 77 & 14.40 & 23 & 1478.0 & 19 & 6604 \\
\hline 90343 & 7.30 & 0.82 & 9.55 & 14 & 1478.0 & 9 & 3691 \\
\hline 101177 & 6.45 & 0.57 & -16.95 & 17 & 1225.7 & 35 & 4036 \\
\hline 109358 & 4.27 & 0.59 & 6.25 & 71 & 75.0 & 24 & 5577 \\
\hline 115404 & 6.59 & 0.93 & 7.60 & 15 & 1410.0 & 11 & 3244 \\
\hline 125184 & 6.47 & 0.72 & -12.40 & 14 & 1475.0 & 11 & 3294 \\
\hline 128165 & 7.23 & 1.00 & 1.25 & 17 & 76.8 & 14 & 4040 \\
\hline 131977 & 5.75 & 1. & 26.85 & 16 & 1476.9 & 29 & 6641 \\
\hline 139323 & 7.60 & & 6730 & 17 & 1477.9 & 13 & 5830 \\
\hline 140538 & 5.86 & 0.68 & .00 & 17 & 77.0 & 24 & 5136 \\
\hline 144579 & 6.66 & 0.73 & -59.45 & 22 & 1477.9 & 264 & 7618 \\
\hline 145742 & 7.57 & 1. & -21.85 & 10 & 1479.0 & 9 & 2991 \\
\hline 151541 & 7.56 & 0.77 & 9.40 & 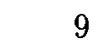 & 6.9 & 9 & 3339 \\
\hline 154345 & 6.77 & 0.73 & -46.95 & 14 & 1479.0 & 16 & 3618 \\
\hline 158633 & 6.43 & 0.76 & -38.60 & 9 & 1477.0 & 23 & 4821 \\
\hline 159222 & 6. & & -51.60 & 12 & .8 & 15 & 3013 \\
\hline 164922 & 6.99 & 80 & 0.15 & 20 & 1414.0 & 17 & 3717 \\
\hline 168009 & 6.29 & 0.64 & -64.65 & 25 & 1479.9 & 17 & 4087 \\
\hline 182488 & 6.37 & 0. & & 15 & & 20 & 3026 \\
\hline 182572 & 5.16 & 0. & -100.35 & 10 & 1182.0 & 330 & 7507 \\
\hline 190007 & 7.46 & 1.13 & -30.40 & 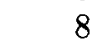 & 1039.1 & 10 & 3272 \\
\hline 190404 & 7.26 & 0.8 & -2.60 & 10 & & 29 & 6972 \\
\hline 193664 & 5.93 & & -4.50 & 11 & 1091.0 & 14 & 4054 \\
\hline 196850 & 6.75 & 0.61 & -21.05 & 14 & 1129.0 & 19 & 4680 \\
\hline 197076 & 6.45 & 0.61 & -35.40 & 10 & 1044.2 & 18 & 4697 \\
\hline 210667 & 7.24 & 0.8 & -19.50 & 13 & 1039.1 & 17 & 2976 \\
\hline 221354 & 6.74 & 0.84 & -25.20 & 10 & 1210.7 & 10 & 2886 \\
\hline
\end{tabular}

\section{Discussion}

Scarfe: Have you enough stars on the ELODIE-corrected CORAVEL list for comparison with the ones we published in 1990 (Scarfe, Batten \& Fletcher, Publ. Dom. Ap. Obs., 18, 21)? I have some additional data for such a comparison. 
Udry: We have not checked yet, but I will do it. All other comparison samples are welcome.

Noyes: I would like to suggest that you add to the list of precise radial-velocity standards published on the Web some brighter dwarfs, in the interests of minimizing time for observing standards.

Udry: The criteria used to define the high-precision radial-velocity standards are strong. It is easy to be a little less severe to include stars with needed characteristics, such as brighter magnitude or higher velocity. 